\title{
Recent advance in detonation theory
}

A detonation wave is a chemical reaction wave propagating at the velocity of a shock wave along the explosive charge. There is great demand for a detonation model that can accurately simulate the detonation process, which would provide a theoretical basis for highly efficient military destruction, the initiation of an atomic bomb, and calculations relating to the supernova SN Ia explosion. Detonation is complicated in that it involves mechanics, chemistry and thermodynamics simultaneously. The detonation product particles move multi-dimensionally, and there are transport effects, such as friction, diffusion and heat transfer, between particles. Because of the difficulty of simulating such complex configurations, classical detonation theory, which employs the ZND model, neglects the transport effects and simplifies detonation as one-dimensional movement. However, experiments have shown that the complex movement and transport effects play important roles in detonation and should be taken into account.

Taking the complex multi-dimensional movement into account, Hu et al. at the Xi'an Modern Chemistry Research Institute proposed the use of the entropy principle to specify the final point of detonation, and the use of the Hamilton principle to describe the complex movement of detonation product particles and determine the real path from explosive to products. A new detonation model named the least-action detonation model (LADM) has been proposed (Sci Sin Phys Mech Astron, 2011, 41: 1230-1238).

The least-action principle is one of the basic principles of nature, from which nearly all physical equations can be derived, including the equations of Newtonian mechanics, relativistic mechanics, electrodynamics, Maxwell's equations, Fermat's principle in optics, and Schrödinger equations in quantum mechanics. In analytical mechanics, the least-action principle is known as Hamilton's principle, which is equivalent to Newton's Law and applies to complex mechanical systems. Hu et al. introduced the Hamilton principle into detonation science to bypass the difficulty of describing the complex movement and transport effects in detonation (Propellants Explos Pyrotech, 2007, 32: 73-79)

Because the LADM model takes complex movement and transport effects into account, the detonation configuration described by the LADM model differs from that described by the ZND model. The flow after the reaction zone in the ZND model is the changing Taylor rarefaction, whereas it is a stationary state in the LADM model. From the displacement of titanium foils embodied in the explosive charge, the movement state of detonation product particles can be judged. The X-ray photograph shows that the titanium foil initially moves $1-2$ $\mathrm{mm}$ because of the moving particles in the reaction zone, and then stops moving and is in a stationary state, which coincides with the prediction of the LADM model.

Much evidence that detonation particles are in a stationary state has already been given in the literature on detonation in the form of data, graphs and photographs. However, such evidence has not been addressed because of its contradiction with the ZND model; moreover, the stationary state has never been considered as the essence of detonation. Blasting models derived for a stationary state have long been used in blasting engineering, but the stationary state has been considered only an assumption because it contradicts the ZND model.

Because the LADM model solves many problems relating to detonation phenomena that cannot be explained by the ZND model, the use of the LADM model also proposes a series of research subjects in detonation science.

In recent years, detonation science has focused on the sub-macroscopic and sub-microscopic structures of detonation phenomena. By contrast, the LADM model emphasizes the general direction decided by the second law of thermodynamics, to grasp the essence of the detonation process.

According to the LADM model in which complex movement and transport effects are taken into account, the detonation path and final point of detonation should differ from those suggested by the ZND model.

Compared with the ZND model, the LADM model incorporates many partial differential equations corresponding to the multiformity of detonation. It is a challenge to solve these partial differential equations, which involve mechanics, chemistry and thermodynamics simultaneously.

Many equations of state have been proposed to calculate the moving Chapman-Jouguet state of detonation products. As theory and experiments show that detonation products are in a stationary state, the establishment of new equations of state becomes an urgent task in the field of detonation science.

"Standard candles" calculations for the supernovae SN Ia explosion have shown that the ZND model is not able to simulate precisely the complex process of detonation and a new detonation model is needed. The LADM model incorporating many partial differential equations is one such candidate model.

See the articles: Hu S M, Tian Q Z, Xiao C, et al. A new detontion model and its examination by experiment (in Chinese). Sci Sin Phys Mech Astron, 2011, 41: $1230-1238$

Hu S M, Li C F, Ma Y H, et al. A detonation model of high/low velocity detonation. Propellants Explos Pyrotech, 2007, 32: $73-79$

Open Access This article is distributed under the terms of the Creative Commons Attribution License which permits any use, distribution, and reproduction in any medium, provided the original author(s) and source are credited. 